\title{
Flows with Slip of Oldroyd-B Fluids over a Moving Plate
}

\author{
Abdul Shakeel, ${ }^{1}$ Sohail Ahmad, ${ }^{2}$ Hamid Khan, ${ }^{1}$ Nehad Ali Shah, ${ }^{3}$ and Sami Ul Haq ${ }^{4}$ \\ ${ }^{1}$ Department of Mathematics, FAST-National University of Computer and Emerging Sciences, Peshawar, \\ Khyber Pakhtunkhwa 25000, Pakistan \\ ${ }^{2}$ Department of Mathematics, COMSATS Institute of Information Technology, Attock, Kamra Road, Punjab 43600, Pakistan \\ ${ }^{3}$ Abdus Salam School of Mathematical Sciences, Government College University, Lahore, Punjab 54000, Pakistan \\ ${ }^{4}$ Department of Mathematics, Islamia College, Peshawar, Khyber Pakhtunkhwa 25000, Pakistan \\ Correspondence should be addressed to Sohail Ahmad; drsohailahmad@ciit-attock.edu.pk
}

Received 12 November 2015; Revised 3 March 2016; Accepted 13 March 2016

Academic Editor: Luigi C. Berselli

Copyright (C) 2016 Abdul Shakeel et al. This is an open access article distributed under the Creative Commons Attribution License, which permits unrestricted use, distribution, and reproduction in any medium, provided the original work is properly cited.

\begin{abstract}
A general investigation has been made and analytic solutions are provided corresponding to the flows of an Oldroyd-B fluid, under the consideration of slip condition at the boundary. The fluid motion is generated by the flat plate which has a translational motion in its plane with a time-dependent velocity. The adequate integral transform approach is employed to find analytic solutions for the velocity field. Solutions for the flows corresponding to Maxwell fluid, second-grade fluid, and Newtonian fluid are also determined in both cases, namely, flows with slip on the boundary and flows with no slip on the boundary, respectively. Some of our results were compared with other results from the literature. The effects of several emerging dimensionless and pertinent parameters on the fluid velocity have been studied theoretically as well as graphically in the paper.
\end{abstract}

\section{Introduction}

Many materials in industry, for instance, grease, polymer melts, drilling mud, clay coating, suspensions, certain oil, and different emulsions, behave in such a way that we cannot describe mathematically through Navier-Stokes equations. For this reason, it is now generally accepted that non-Newtonian fluid models are more appropriate than Newtonian ones and, in practical applications, the behavior of nonNewtonian fluids cannot be replaced with that of Newtonian fluids. Therefore, the study of non-Newtonian fluids has become very important due to their large number of applications in industry.

The reader can see [1] for the latest and complete discussion on Oldroyd-B fluid models. To the best of the authors' knowledge, the first exact solutions corresponding to these fluid models seem to be those obtained by Tanner [2]. Furthermore, some other useful as well as simpler solutions can be found in [3] regarding the study of Oldroyd-B fluids.

In the above studies, the effect of fluid slippage is not considered. The flow of fluids induced by a motion of a plate is called Stokes flow. Solution for some Stokes flows in different geometries and under the assumption of no-slip boundary condition can be found in [4-7].

However, the no-slip condition is inadequate in several situations, for instance, mechanics of thin fluids, problems having multiple interfaces, microchannel flows, flows in wavy tubes, and flows of polymeric liquids with high molecular weight [8].

It is vital to study the effect of fluid slippage as it finds many applications in industry. When a surface moves, the slip is mainly produced by the roughness of surface and rarefaction of the fluid and the velocity on the surface. Navier [9] proposed slip boundary condition in which it was stated that the velocity of the fluid depends on the shear stress. For describing the slip that occurs at solid boundaries, a large number of models have been proposed [10]. Other studies of slip at the boundary can be seen in [11-14]. Some non-Newtonian fluids, such as polymer melts, often exhibit macroscopic wall slip, which is generally described by a nonlinear relation between wall slip velocity and the friction at the wall.

The aim of the present communication is to study Stokes flows of an Oldroyd-B fluid on a flat plate under the slip 
boundary conditions assumption between the plate and the fluid. The motion of the plate is a rectilinear translation in its plane. Exact expressions for the velocity are determined by means of the Laplace transforms. Expressions for the relative velocity are also determined and the solutions corresponding to flows with no slip at the boundary are presented. The particular case, namely, sine oscillations of the wall, is studied. Some relevant properties of the velocity and comparisons between solutions with slip and no slip at the boundary are presented by using graphical illustrations generated by the software Mathcad.

\section{Mathematical Formulation of the Problem}

We consider an incompressible Oldroyd-B fluid occupying the space over an infinite plate which is situated in the $(x, z)$ plane of the Cartesian coordinate system with the positive $y$ axis in the upward direction. Initially, both the fluid and the plate are at rest. At $t=0^{+}$the fluid is set in motion by the plate, which begins to move along the $x$-axis. The velocity of the plate is assumed to be of the form $U_{o} f(t)$, where $U_{o}>0$ is a constant and $f(t)$ is a piecewise continuous dimensionless function defined on $[0, \infty)$ and $f(0)=0$. Furthermore we suppose that the Laplace transform of the function $f$ exists. In the case of parallel flow along the $x$-axis, the velocity vector is $\boldsymbol{v}=(u(y, t), 0,0)$ whereas [15-17] lead us to the following governing equation:

$$
\lambda \frac{\partial^{2} u(y, t)}{\partial t^{2}}+\frac{\partial u(y, t)}{\partial t}=v\left(1+\lambda_{r} \frac{\partial}{\partial t}\right) \frac{\partial^{2} u(y, t)}{\partial y^{2}}
$$

where $\lambda$ is the relaxation time, $\lambda_{r}$ is the retardation time, $\nu=$ $\mu / \rho$ is the kinematic viscosity, $\mu$ is the dynamic viscosity, and $\rho$ is the constant density of the fluid. In this work we consider the existence of slip at the wall and assume that the relative velocity between the velocity of the fluid at the wall $u(0, t)$ and the speed of the wall is proportional to the shear rate at the wall $[18,19]$. The adequate initial and boundary conditions are given by

$$
\begin{aligned}
u(0, t)-\beta \frac{\partial u(0, t)}{\partial y} & =U_{o} f(t), \quad \beta \geq 0, t>0, \\
u(y, 0) & =\frac{\partial u(y, 0)}{\partial t}=0, \quad y \geq 0, \\
u(y, t) & \longrightarrow 0 \quad \text { for } y \longrightarrow \infty
\end{aligned}
$$

where $\beta$ is the slip coefficient.

By using the characteristic time $T$, introducing the following nondimensional quantities to (1) and (2),

$$
\begin{aligned}
t^{*} & =\frac{t}{T} ; \quad T>0, \\
y^{*} & =\frac{y}{U_{o} T},
\end{aligned}
$$

$$
\begin{aligned}
u^{*} & =\frac{u}{U_{o}}, \\
\lambda^{*} & =\frac{\lambda}{T}, \\
\lambda_{r}^{*} & =\frac{\lambda_{r}}{T}, \\
\beta^{*} & =\frac{\beta}{U_{o} T}, \\
g\left(t^{*}\right) & =f\left(T t^{*}\right),
\end{aligned}
$$

and dropping out the $*$ notation we get the following nondimensional problem:

$$
\lambda \frac{\partial^{2} u(y, t)}{\partial t^{2}}+\frac{\partial u(y, t)}{\partial t}=\frac{1}{\operatorname{Re}}\left(1+\lambda_{r} \frac{\partial}{\partial t}\right) \frac{\partial^{2} u(y, t)}{\partial y^{2}} .
$$

The nondimensional initial and boundary conditions are

$$
\begin{aligned}
u(0, t)-\beta \frac{\partial u(0, t)}{\partial y} & =g(t), \text { for } t>0, \\
u(y, 0) & =\frac{\partial u(y, 0)}{\partial t}=0, \text { for } y>0, \\
u(y, t) & \longrightarrow 0, \text { for } y \rightarrow \infty
\end{aligned}
$$

with $\operatorname{Re}=U_{o}^{2} T / \nu$, the Reynolds number.

\section{Calculations for Velocity Field}

3.1. Oldroyd-B Fluid with Slip at the Wall. Applying Laplace transform to (4), (5a), and (5c) and using (5b), we obtain the transformed problem:

$$
\begin{aligned}
\frac{\partial^{2} \bar{u}(y, q)}{\partial y^{2}}-\frac{\operatorname{Re}\left(\lambda q^{2}+q\right)}{\left(1+\lambda_{r} q\right)} \bar{u}(y, q) & =0, \\
\bar{u}(0, q)-\beta \frac{\partial \bar{u}(0, q)}{\partial y} & =G(q), \\
\bar{u}(y, q) & \longrightarrow 0, \quad y \rightarrow \infty .
\end{aligned}
$$

The solution of the set of (6)-(8) is given by

$$
\bar{u}(y, q)=F(y, q) \cdot G(q)
$$

where

$$
\begin{gathered}
F(y, q)=\frac{b \exp \left(-a \sqrt{\left(\lambda q^{2}+q\right) /\left(\lambda_{r} q+1\right)}\right)}{b+\sqrt{\left(\lambda q^{2}+q\right) /\left(\lambda_{r} q+1\right)}}, \\
a=y \sqrt{\operatorname{Re}}, b=\frac{1}{\beta \sqrt{\mathrm{Re}}}
\end{gathered}
$$

and $G(q)=L\{g(t)\}$. 
In order to obtain the inverse Laplace transform of function $\bar{u}(y, q)$, we consider the auxiliary functions

$$
\begin{aligned}
F_{1}(y, q) & =\frac{b \exp (-a \sqrt{q})}{b+\sqrt{q}}, \\
w(q) & =\frac{\lambda q^{2}+q}{\lambda_{r} q+1} .
\end{aligned}
$$

Seeing that $F(y, q)=F_{1}[y, w(q)]$, we have

$$
f(y, t)=L^{-1}\{F(y, q)\}=\int_{0}^{\infty} f_{1}(y, x) h(x, t) d x,
$$

where

$$
\begin{aligned}
f_{1}(y, t) & =L^{-1}\left\{F_{1}(y, q)\right\} \\
= & \frac{e^{-y^{2} \operatorname{Re} / 4 t}}{\beta \sqrt{\operatorname{Re} \pi t}} \\
& \quad-\frac{1}{\beta^{2} \operatorname{Re}} e^{y / \beta+t / \beta^{2} \operatorname{Re}} \operatorname{Erfc}\left(\frac{y \sqrt{\operatorname{Re}}}{2 \sqrt{t}}+\frac{1}{\beta} \sqrt{\frac{t}{\operatorname{Re}}}\right),
\end{aligned}
$$

and $h(x, t)=L^{-1}\{\exp (-x w(q))\}$ is given by

$$
\begin{aligned}
h(x, t)= & \exp (\gamma x) \delta\left(t-\frac{\lambda x}{\lambda_{r}}\right)-\sqrt{\frac{\gamma x}{\lambda_{r} t-\lambda x}} \\
& \cdot J_{1}\left(\frac{2}{\lambda_{r}} \sqrt{\gamma x\left(\lambda_{r} t-\lambda x\right)}\right) \\
& \cdot \exp \left(\frac{\left(2 \lambda-\lambda_{r}\right) x-\lambda_{r} t}{\lambda_{r}{ }^{2}}\right),
\end{aligned}
$$

with $\gamma=\left(\lambda-\lambda_{r}\right) / \lambda_{r}^{2}$.

Now, introducing $f_{1}(y, t)$ and $h(x, t)$ into (12), we obtain

$$
\begin{aligned}
& f(y, t)=\frac{b \sqrt{\lambda_{r}}}{\sqrt{\lambda \pi t}} \exp \left(-\frac{\lambda \operatorname{Re} y^{2}}{4 \lambda_{r} t}+\frac{\gamma \lambda_{r}}{\lambda} t\right)-\frac{\lambda_{r} b^{2}}{\lambda} \\
& \cdot \exp \left(y b \sqrt{\operatorname{Re}}+\frac{b^{2}+\gamma}{\lambda} \lambda_{r} t\right) \operatorname{Erfc}\left(\frac{y \sqrt{\lambda \operatorname{Re}}}{2 \sqrt{\lambda_{r} t}}\right. \\
& \left.+b \sqrt{\frac{\lambda_{r} t}{\lambda}}\right)+\int_{0}^{\infty}\left[\frac{b}{\sqrt{\pi x}}\right. \\
& \cdot \exp \left(-\frac{y^{2} \operatorname{Re}}{4 x}+\frac{\left(2 \lambda-\lambda_{r}\right) x-\lambda_{r} t}{\lambda_{r}{ }^{2}}\right) \\
& -b^{2} \exp \left(y b \sqrt{\operatorname{Re}}+b^{2} x+\frac{\left(2 \lambda-\lambda_{r}\right) x-\lambda_{r} t}{\lambda_{r}{ }^{2}}\right) \\
& \left.\cdot \operatorname{Erfc}\left(\frac{y \sqrt{\operatorname{Re}}}{2 \sqrt{x}}+b \sqrt{x}\right)\right] \times \sqrt{\frac{\gamma x}{\lambda_{r} t-\lambda x}} \cdot J_{1}\left(\frac{2}{\lambda_{r}}\right. \\
& \left.\cdot \sqrt{\gamma x\left(\lambda_{r} t-\lambda x\right)}\right) d x,
\end{aligned}
$$

and the velocity field corresponding to the flow, with slip at the wall of an Oldroyd-B fluid, is given by

$$
u_{s}(y, t)=(f * g)(t)=\int_{0}^{t} f(y, s) g(t-s) d s,
$$

where $f(y, t)$ is given by (15).

3.2. Oldroyd-B Fluid with No Slip at the Wall. In this particular case, function $F(y, q)$ given by (10) becomes

$$
F_{\text {ns }}(y, q)=\exp \left(-y \sqrt{\operatorname{Re}} \sqrt{\frac{\lambda q^{2}+q}{\lambda_{r} q+1}}\right) .
$$

By using the auxiliary functions,

$$
\begin{aligned}
& F_{1 \mathrm{~ns}}(y, q)=\exp (-y \sqrt{\operatorname{Re} q}), \\
& f_{1 \mathrm{~ns}}(y, t)=\frac{y \sqrt{\operatorname{Re}}}{2 t \sqrt{\pi t}} \exp \left(-\frac{y^{2} \operatorname{Re}}{4 t}\right),
\end{aligned}
$$

together with the relation

$$
\begin{gathered}
f_{\mathrm{ns}}(y, t)=L^{-1}\left\{F_{\mathrm{ns}}(y, q)\right\}=\int_{0}^{\infty} f_{1 \mathrm{~ns}}(y, x) \\
\cdot h(x, t) d x=\frac{y \sqrt{\operatorname{Re} \lambda}}{2 \sqrt{\pi \lambda_{r}}} t^{-3 / 2} \exp \left(\frac{\gamma \lambda_{r}}{\lambda} t\right. \\
\left.-\frac{y^{2} \operatorname{Re} \lambda}{4 \lambda_{r} t}\right)-\frac{y \sqrt{\operatorname{Re} \gamma}}{2 \sqrt{\pi}} \\
\cdot \int_{0}^{\infty} e^{-y^{2} \operatorname{Re} / 4 x+\left(\left(2 \lambda-\lambda_{r}\right) x-\lambda_{r} t\right) / \lambda_{r}{ }^{2}} \\
\cdot \frac{1}{x \sqrt{\lambda_{r} t-\lambda x}} J_{1}\left(\frac{2}{\lambda_{r}} \sqrt{\gamma x\left(\lambda_{r} t-\lambda x\right)}\right) d x .
\end{gathered}
$$

The velocity field corresponding to the flow with no-slip conditions of the Oldroyd-B fluid is given by

$$
\begin{aligned}
& u_{\mathrm{ns}}(y, t)=\int_{0}^{t} f_{\mathrm{ns}}(y, s) g(t-s) d s=\frac{2}{\sqrt{\pi}} \\
& \cdot \int_{y \sqrt{\operatorname{Re} \lambda} / 2 \sqrt{\lambda_{r} t}}^{\infty} e^{-z^{2}+\gamma \operatorname{Re} y^{2} / 4 z^{2}} g\left(t-\frac{\lambda \operatorname{Re} y^{2}}{4 \lambda_{r} z^{2}}\right) d z \\
& -\frac{y \sqrt{\operatorname{Re} \gamma}}{2 \sqrt{\pi}} \\
& \cdot \int_{0}^{\infty} \int_{0}^{t} g(t-s) \frac{e^{-y^{2} \operatorname{Re} / 4 x+\left(\left(2 \lambda-\lambda_{r}\right) x-\lambda_{r} s\right) / \lambda_{r}{ }^{2}}}{x \sqrt{\lambda_{r} s-\lambda x}} \\
& \quad \times J_{1}\left(\frac{2}{\lambda_{r}} \sqrt{\gamma x\left(\lambda_{r} s-\lambda x\right)}\right) d s d x .
\end{aligned}
$$


It is important to point out that the solution given by (20) is similar to that obtained recently by Fetecau et al. [20, Eq. (4.28)].

3.3. Maxwell Fluid with Slip Condition. For the flows of Maxwell fluids with slip condition at the wall, (10) becomes

$$
\begin{aligned}
F_{\text {sM }}(y, q) & \\
= & \frac{(b / \sqrt{2}) \exp \left(-y \sqrt{\lambda \operatorname{Re}} \sqrt{(q+1 / 2 \lambda)^{2}-(1 / 2 \lambda)^{2}}\right)}{b / \sqrt{2}+\sqrt{(q+1 / 2 \lambda)^{2}-(1 / 2 \lambda)^{2}}} \\
= & b_{1} \exp \frac{\left(-y \sqrt{\lambda \operatorname{Re}} \sqrt{\left(q+a_{1}\right)^{2}-a_{1}^{2}}\right)}{b_{1}} \\
& +\sqrt{\left(q+a_{1}\right)^{2}-a_{1}^{2}}=F_{1}\left(y, \sqrt{w_{1}(q)}\right),
\end{aligned}
$$

where $F_{1}(\cdot, \cdot)$ is given by $(11)$ and $w_{1}(q)=\left(q+a_{1}\right)^{2}-a_{1}^{2}, b_{1}=$ $b / \sqrt{\lambda}$, and $a_{1}=1 / 2 \lambda$.

The inverse Laplace transform of function (21) is

$$
\begin{aligned}
& f_{\mathrm{sm}}(y, t)=\left[f_{1}(y, t)\right. \\
& \left.+a_{1} \int_{0}^{t} f_{1}\left(y_{1} \sqrt{t^{2}-u^{2}}\right) I_{1}(a, u) d u\right] e^{-a_{1} t} \\
& . \frac{b_{1} e^{-y^{2} \operatorname{Re} \lambda / 4 t-a_{1} t}}{\sqrt{\pi t}} \\
& -b_{1}^{2} e^{y b_{1} \sqrt{\operatorname{Re} \lambda}+b_{1}^{2} t-a_{1} t} \operatorname{Erfc}\left(\frac{y \sqrt{\operatorname{Re} \lambda}}{2 \sqrt{t}}+b \sqrt{t}\right) \\
& +a_{1} e^{-a_{1} t} \int_{0}^{t}\left[\frac{b_{1} e^{-y^{2} \operatorname{Re} \lambda / 4 z-a_{1} z}}{\sqrt{\pi z}}\right. \\
& \left.-b_{1}^{2} e^{y b_{1} \sqrt{\operatorname{Re} \lambda}+b_{1}^{2}-a_{1} z} \operatorname{Erfc}\left(\frac{y \sqrt{\operatorname{Re} \lambda}}{2 \sqrt{z}}+b_{1} \sqrt{z}\right)\right] \\
& . \frac{z}{\sqrt{t^{2}-z^{2}}} I_{1}\left(a_{1} \sqrt{t^{2}-z^{2}}\right) d z
\end{aligned}
$$

and the velocity field is given by

$$
u_{\mathrm{sM}}(y, t)=(f * g)(t)=\int_{0}^{t} f_{\mathrm{SM}}(y, s) g(t-s) d s
$$

3.4. Maxwell Fluid with No Slip at the Wall. Making $\beta \rightarrow 0$ and therefore $b \rightarrow 0$ into (21) or $\lambda_{r} \rightarrow 0$ into (17), we have

$$
\begin{aligned}
F_{\mathrm{nsM}}(y, q) & =\exp \left(-y \sqrt{\operatorname{Re}} \sqrt{\lambda q^{2}+q}\right) \\
& =\exp \left(-y \sqrt{\operatorname{Re} \lambda} \sqrt{\left(q+a_{1}\right)^{2}-a_{1}^{2}}\right) .
\end{aligned}
$$

Using the formula

$$
L^{-1}\left\{e^{-y \sqrt{\operatorname{Re} \lambda} \sqrt{q}}\right\}=h_{1}(y, t)=\frac{y \sqrt{\operatorname{Re} \lambda} e^{-y^{2} \operatorname{Re} \lambda / 4 t}}{2 t \sqrt{\pi t}}
$$

we obtain

$$
\begin{aligned}
& f_{\mathrm{nsM}}(y, t)=\left[h_{1}(y, t)\right. \\
& \left.+a_{1} \int_{0}^{t} h_{1}(y, z) \frac{z}{\sqrt{t^{2}-z^{2}}} I_{1}\left(a_{1} \sqrt{t^{2}-z^{2}}\right) d z\right] \\
& \cdot e^{-a_{1} t}=\frac{y \sqrt{\operatorname{Re} \lambda}}{2 t \sqrt{\pi t}} e^{-y^{2} \operatorname{Re} \lambda / 4 t-a_{1} t} \\
& +a_{1} e^{-a_{1} t} \int_{0}^{t} \frac{y \sqrt{\operatorname{Re} \lambda}}{2 z \sqrt{\pi z}} e^{-y^{2} \operatorname{Re} \lambda / 4 z} \\
& \cdot \frac{z}{\sqrt{t^{2}-z^{2}}} I_{1}\left(a_{1} \sqrt{t^{2}-z^{2}}\right) d z
\end{aligned}
$$

and the velocity field

$$
\begin{aligned}
& u_{\mathrm{nsM}}(y, t)=(f * g)(t)=\int_{0}^{t} f_{\mathrm{nsM}}(y, s) g(t-s) d s \\
& =\frac{2}{\sqrt{\pi}} \int_{y \sqrt{\operatorname{Re} \lambda} / 2 \sqrt{t}}^{\infty} e^{-z^{2}-a_{1} \lambda \operatorname{Re} y^{2} / 4 z^{2}} g(t \\
& \left.-\frac{\lambda \operatorname{Re} y^{2}}{4 z^{2}}\right) d z+a_{1} \int_{0}^{t} \int_{0}^{s} g(t-s) \\
& . \frac{y \sqrt{\operatorname{Re} \lambda}}{2 z \sqrt{\pi z}} e^{-y^{2} \operatorname{Re} \lambda / 4 z-a_{1} s} \\
& \cdot \frac{z}{\sqrt{s^{2}-z^{2}}} I_{1}\left(a_{1} \sqrt{s^{2}-z^{2}}\right) d s d z .
\end{aligned}
$$

3.5. Second-Grade Fluid with Slip at the Wall. Making $\lambda \rightarrow 0$ into (10) and (14) and using the results

$$
\begin{aligned}
M_{1} & =\frac{b}{\sqrt{\pi}} \int_{0}^{\infty} e^{-y^{2} \mathrm{Re} / 4 x-x / \lambda_{r}} \frac{d x}{\sqrt{x}} \\
& =\frac{b}{\sqrt{\pi}} \int_{0}^{\infty} e^{\left(-y^{2} \mathrm{Re} / \lambda_{r}\right) / 4 z^{2}-z^{2}} 2 \sqrt{\lambda_{r}} d z \\
& =b \sqrt{\lambda_{r}} \frac{2}{\sqrt{\pi}} \int_{0}^{\infty} e^{-z^{2}-\left(y \sqrt{\operatorname{Re}} / \lambda_{r}\right)^{2} / 4 z^{2}} d z \\
& =b \sqrt{\lambda_{r}} \frac{2}{\sqrt{\pi}} \cdot \frac{\sqrt{\pi}}{2} e^{-1 \cdot y \sqrt{\operatorname{Re}} / \sqrt{\lambda_{r}}}=b \sqrt{\lambda_{r}} e^{-y \sqrt{\operatorname{Re}} / \sqrt{\lambda_{r}}}
\end{aligned}
$$


we obtain the velocity field of the second-grade fluid with slip at the wall in the equivalent forms

$$
\begin{gathered}
u_{\mathrm{sSG}}(y, t)=b \sqrt{\lambda_{r}} e^{-y \sqrt{\operatorname{Re}} / \sqrt{\lambda_{r}}} g(t)-b^{2} e^{b y \sqrt{\operatorname{Re}}} g(t) \\
\cdot \int_{0}^{\infty} e^{b^{2} x-x / \lambda_{r}} \operatorname{Erfc}\left(\frac{y \sqrt{\operatorname{Re}}}{2 \sqrt{x}}+b \sqrt{x}\right) d x+\frac{1}{\lambda_{r}} \\
\cdot \int_{0}^{\infty}\left[\frac{b e^{-y^{2} \operatorname{Re} / 4 x}}{\sqrt{\pi x}}\right. \\
\left.-b^{2} e^{y b \sqrt{\operatorname{Re}}+b^{2} x} \operatorname{Erfc}\left(\frac{y \sqrt{\operatorname{Re}}}{2 \sqrt{x}}+b \sqrt{x}\right)\right] \\
\cdot e^{(-x+t) / \lambda_{r}} \sqrt{\frac{x}{t}} I_{1}\left(\frac{2}{\lambda_{r}} \sqrt{x t}\right) d x
\end{gathered}
$$

or

$$
\begin{aligned}
& u_{\mathrm{sSG}}(y, t)=b\left(b^{2} \sqrt{\lambda_{r}}-b+\sqrt{\lambda_{r}}\right) e^{-y \sqrt{\operatorname{Re}} / \sqrt{\lambda_{r}}} g(t) \\
& +\frac{e^{-t / \lambda_{r}}}{\sqrt{\lambda_{r} t}} g(t) \times \int_{0}^{\infty}\left[\frac{b e^{-y^{2} \operatorname{Re} / 4 x-x / \lambda_{r}}}{\sqrt{\pi x}}\right. \\
& \left.-b^{2} e^{b y \sqrt{\operatorname{Re}}+b^{2} x-x / \lambda_{r}} \operatorname{Erfc}\left(\frac{y \sqrt{\mathrm{Re}}}{2 \sqrt{x}}+b \sqrt{x}\right)\right] \\
& \cdot \sqrt{x} I_{1}\left(\frac{2}{\sqrt{\lambda_{r}}} \sqrt{x t}\right) d x .
\end{aligned}
$$

3.6. Second-Grade Fluid with No-Slip Condition. Making $\lambda \rightarrow$ 0 into (17) and using (18) and (28) we have

$$
\begin{aligned}
& u_{\mathrm{nsSG}}(y, t)=e^{-y \sqrt{\mathrm{Re} / \lambda_{r}}} g(t)+\frac{y \sqrt{\mathrm{Re}}}{2 \lambda_{r} \sqrt{\pi}} \\
& \cdot \int_{0}^{\infty} \int_{0}^{t} \frac{g(t-s)}{x \sqrt{s}} e^{-y^{2} \operatorname{Re} / 4 x-x / \lambda_{r}-s / \lambda_{r}} I_{1}\left(\frac{2}{\lambda_{r}}\right. \\
& \cdot \sqrt{x s}) d s d x .
\end{aligned}
$$

3.7. Newtonian Fluid with/without Slip Condition. These cases are obtained easily from (6) and (10) by making $\lambda=$ $\lambda_{r}=0$. We obtain the following expressions for the velocity field:

$$
\begin{gathered}
u_{\mathrm{sN}}(y, t)=\int_{0}^{t} g(t-\tau) v_{\mathrm{sN}}(y, \tau) d \tau, \\
u_{\mathrm{nsN}}(y, t)=\int_{0}^{t} g(t-\tau) v_{\mathrm{nsN}}(y, \tau) d \tau,
\end{gathered}
$$

where

$$
\begin{aligned}
v_{\mathrm{sN}}(y, t)= & \frac{1}{\beta \sqrt{\mathrm{Re}}} \frac{1}{\sqrt{\pi t}} \exp \left(\frac{-y^{2} \mathrm{Re}}{4 t}\right)-\left(\frac{1}{\beta \sqrt{\mathrm{Re}}}\right)^{2} \\
& \cdot \exp \left(\frac{y}{\beta}+\frac{t}{\beta^{2} \mathrm{Re}}\right) \\
& \cdot \operatorname{erfc}\left(\frac{y \sqrt{\mathrm{Re}}}{2 \sqrt{t}}+\frac{\sqrt{t}}{\beta \sqrt{\mathrm{Re}}}\right),
\end{aligned}
$$

for flows with slip on the boundary, and

$$
v_{\mathrm{nsN}}(y, t)=\frac{y \sqrt{\mathrm{Re}}}{2 t \sqrt{\pi t}} \exp \left(\frac{-y^{2} \mathrm{Re}}{4 t}\right),
$$

for flows with no slip on the boundary, respectively.

We mention that the results given by (33) and (34) are the same as those obtained by Fetecau et al. (see [21], Eq. (29), for $M=0, \gamma$ replaced by $\beta \sqrt{\mathrm{Re}}$ and $y$ replaced by $y \sqrt{\mathrm{Re}})$.

Also, if in our results we consider $g(t)=\sin (\omega t)$ or $g(t)=$ $\cos (\omega t),(32)-(34)$ become equivalent to the results of Khaled and Vafai (see [22], Eqs. (8), (9), (10), (16)) and to the results obtained by Hayat et al. (see [23], Eqs. (13), (14), with $M=0$ and $K \rightarrow \infty$ ).

In the case of no slip on the boundary, our solution (32) together with (34) is identical with the result obtained by Fetecau et al. (see [24], Eq. (31) with $K_{\text {eff }}=0$ ).

\section{Numerical Results and Conclusions}

In this paper we have studied the flow of Oldroyd-B fluids generated by a moving flat plate. Using Laplace transform method, we obtained analytical expressions of the velocity for both cases of flows with slip at the boundary and without slip on the boundary. The plate velocity was considered in a general form $U_{0} f(t)$; therefore, solutions for several practical problems can be obtained by choosing suitable forms of the function $f(t)$. From the dimensionless form of the studied problem it can be seen that only Reynolds number and nondimensional relaxation and retardation time influence the fluid flows. From this reason, the numerical studies are made for several values of Reynolds number and of the time $t$. Graphs of velocity were plotted versus spatial coordinate $y$ for the case of translation of the plate with constant velocity, namely, for $f(t)=1$. Velocity fields corresponding to flows of Maxwell fluid, second-grade fluid, and Newtonian fluid were also determined, in both cases, namely, flows with slip on the boundary and flows with no slip on the boundary. In order to study the physical behavior of the fluid, some numerical simulations were made using the Mathcad software. In Figures 1 and 2, curves corresponding to velocity of the Oldroyd$\mathrm{B}$ fluid for $\lambda=0.25$ and $\lambda_{r}=0.15$ and the dimensionless friction coefficient $\beta=0.75$ are sketched. In Figures 1 and 2 , the velocity curves for three values of time $t$ and for three values of Reynolds number Re in the case of flow with slip at the plate and in the case of no slip at the plate are plotted. 

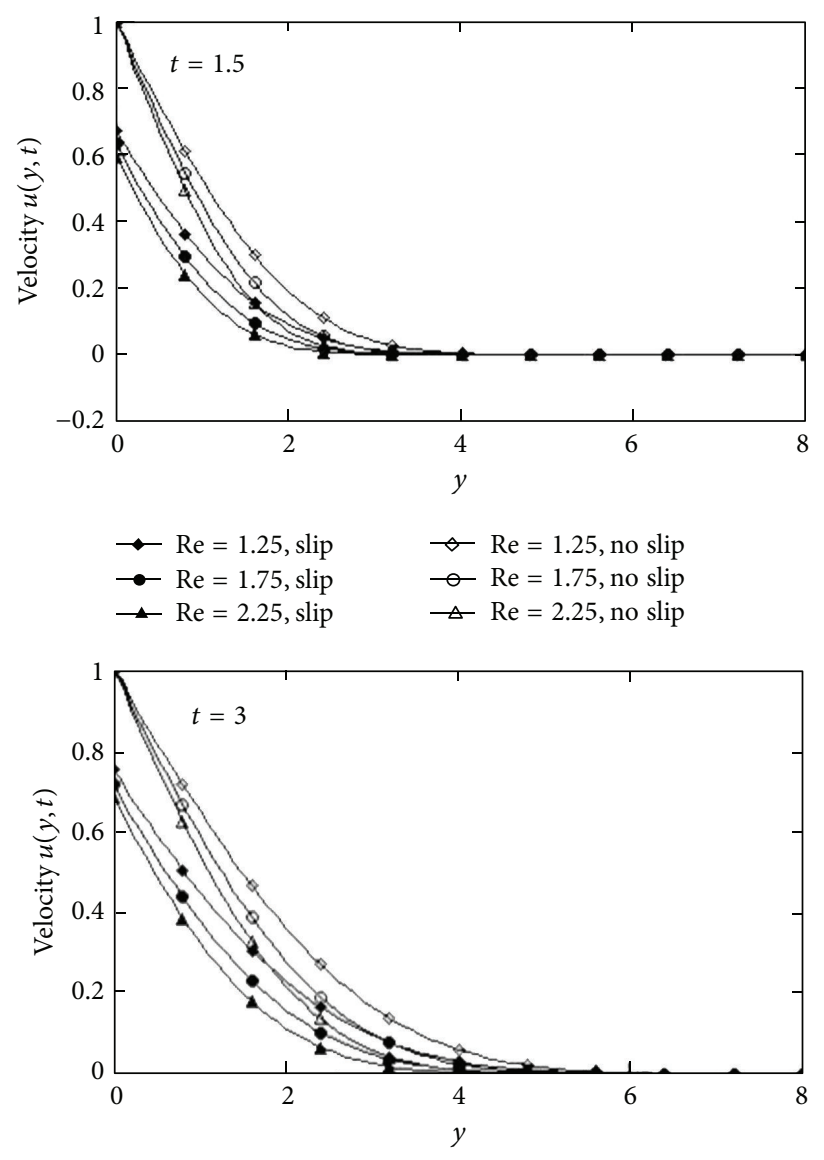

$$
\begin{array}{ll}
\multimap \operatorname{Re}=1.25 \text {, slip } & \multimap \operatorname{Re}=1.25 \text {, no slip } \\
\multimap \operatorname{Re}=1.75 \text {, slip } & \multimap \mathrm{Re}=1.75 \text {, no slip } \\
\multimap \operatorname{Re}=2.25 \text {, slip } & \multimap \mathrm{Re}=2.25 \text {, no slip }
\end{array}
$$



$$
\begin{array}{ll}
\multimap \operatorname{Re}=1.25 \text {, slip } & \diamond \mathrm{Re}=1.25 \text {, no slip } \\
\multimap \mathrm{Re}=1.75 \text {, slip } & \multimap \mathrm{Re}=1.75 \text {, no slip } \\
\multimap \mathrm{Re}=2.25 \text {, slip } & \multimap \mathrm{Re}=2.25 \text {, no slip }
\end{array}
$$

FIGURE 1: Velocity profiles of Oldroyd-B fluid for slip condition (16) with $\beta=0.75$ and no-slip condition $(20)$ for $\lambda=0.25, \lambda_{r}=0.15$, and different Reynolds number.

For a fixed value of the time $t$ or for a fixed value of the Reynolds number the issues which must be highlighted are as follows.

The fluid flows more slowly if slippage occurs on the boundary. Increasing of the Reynolds number values leads to slowing of the flows in both cases, with or without slip. If the values of the time $t$ are increasing, then it increases the thickness of the velocity boundary layer.

Figure 3 was drawn in order to compare the velocity flows for Oldroyd-B, Maxwell, and Newtonian fluids. It is 

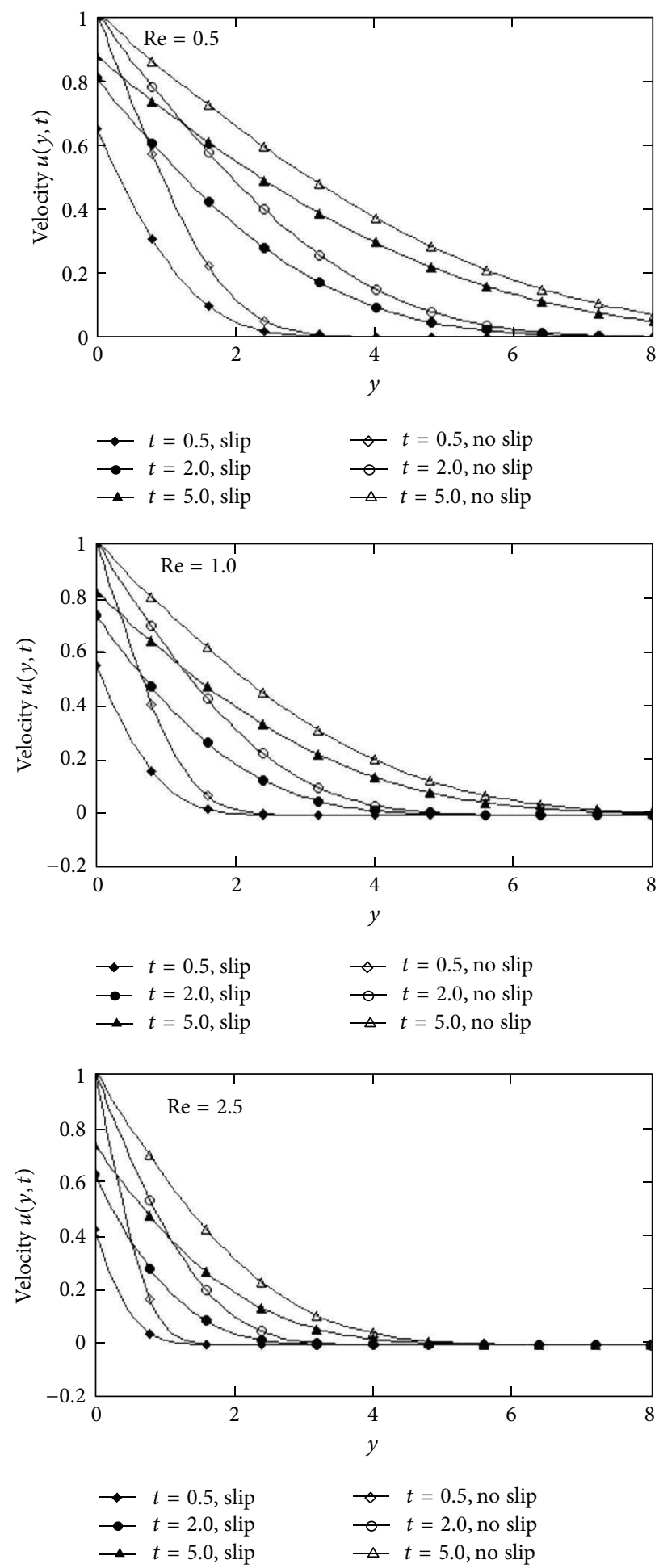

FIGURE 2: Velocity profiles of Oldroyd-B fluid for slip condition (16) with $\beta=0.75$ and no-slip condition $(20)$ for $\lambda=0.25, \lambda_{r}=0.15$, and different values of the time $t$.

important to note that, for the flow with no slip on the boundary, the velocity of Maxwell fluid has significant variations in the area near plate. This no longer occurs if the flow is with slip at the boundary. Also, the thickness of the boundary layer of the Maxwell fluid is the smallest and the speed of this type of fluid becomes zero more quickly than other fluids. If the values of the time $t$ are increasing, then the differences between velocities of the three fluids become insignificant. 


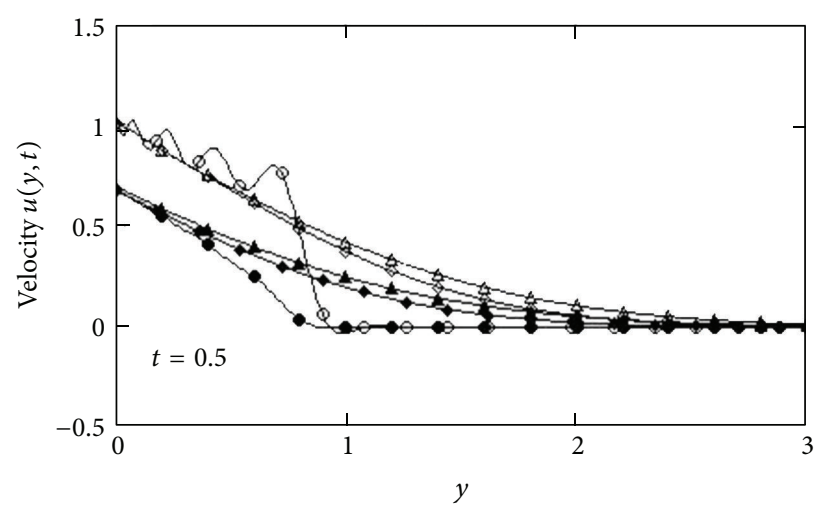

$\rightarrow$ Oldroyd-B fluid, slip $\rightarrow$ Oldroyd-B fluid, no slip

- Maxwell fluid, slip $\quad$ - Maxwell fluid, no slip

$\_$Newtonian fluid, slip $\triangle$ Newtonian fluid, no slip

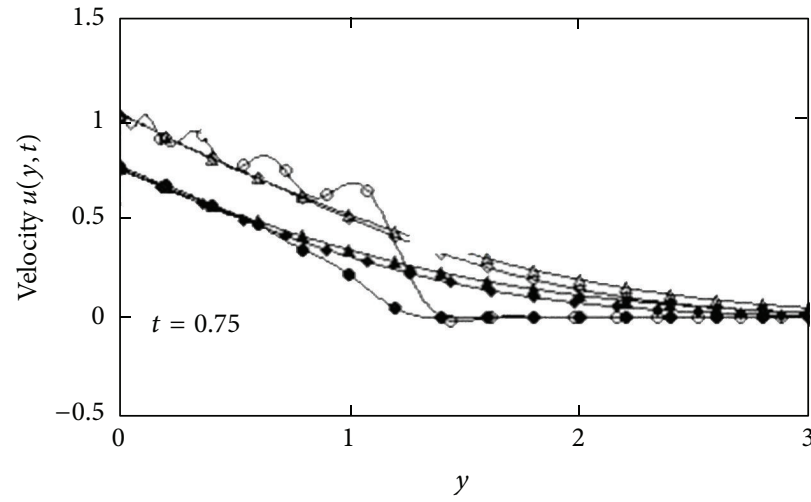

$\rightarrow$ Oldroyd-B fluid, slip $\rightarrow$ Oldroyd-B fluid, no slip

$\rightarrow$ Maxwell fluid, slip $\quad \multimap$ Maxwell fluid, no slip

$\_$Newtonian fluid, slip $\triangle$ Newtonian fluid, no slip

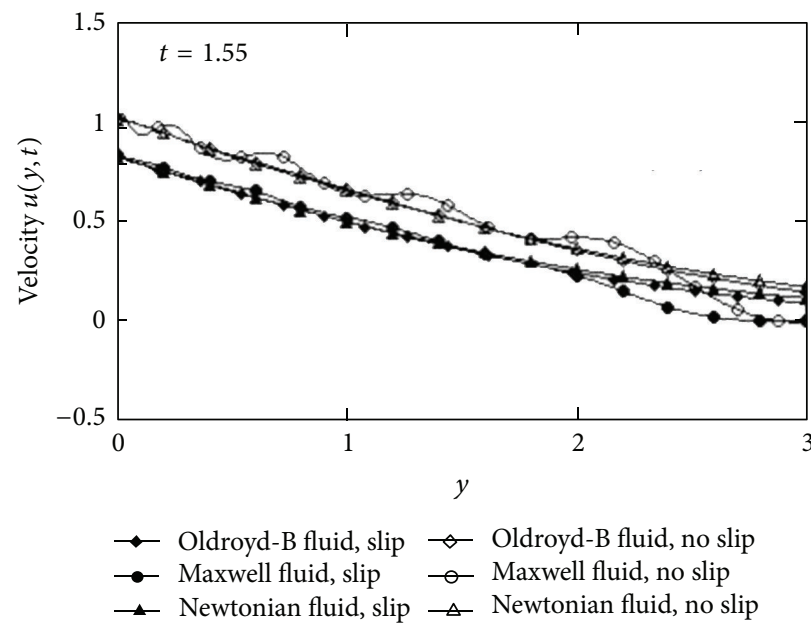

FIGURE 3: Comparison between velocity profiles of three fluid models with slip condition with $\beta=0.55$ and no-slip condition, for Re $=0.65$, $\lambda=0.55$, and $\lambda_{r}=0.35$. 


\section{Competing Interests}

The authors declare that they have no competing interests.

\section{References}

[1] K. R. Rajagopal and A. R. Srinivasa, "A thermodynamic frame work for rate type fluid models," Journal of Non-Newtonian Fluid Mechanics, vol. 88, no. 3, pp. 207-227, 2000.

[2] R. I. Tanner, "Note on the Rayleigh problem for a visco-elastic fluid," Zeitschrift für Angewandte Mathematik und Physik, vol. 13, no. 6, pp. 573-580, 1962.

[3] K. R. Rajagopal and R. K. Bhatnagar, "Exact solutions for some simple flows of an Oldroyd-B fluid," Acta Mechanica, vol. 113, no. 1, pp. 233-239, 1995.

[4] N. D. Waters and M. J. King, "Unsteady flow of an elasticoviscous liquid," Rheologica Acta, vol. 9, no. 3, pp. 345-355, 1970.

[5] P. Puri and P. K. Kythe, "Stokes first and second problems for Rivlin-Ericksen fluids with neoclassical heat condition," ASME Journal of Heat Transfer, vol. 120, pp. 44-50, 1996.

[6] P. M. Jordan and A. Puri, "Revisiting Stokes' first problem for Maxwell fluids," The Quarterly Journal of Mechanics and Applied Mathematics, vol. 58, no. 2, pp. 213-227, 2005.

[7] C. Fetecau, D. Vieru, and C. Fetecau, "A note on the second problem of Stokes for Newtonian fluids," International Journal of Non-Linear Mechanics, vol. 43, no. 5, pp. 451-457, 2008.

[8] R. I. Tanner, "Partial wall slip in polymer flow," Industrial and Engineering Chemistry Research, vol. 33, no. 10, pp. 2434-2436, 1994.

[9] M. Navier, "Mémoire sur les lois du mouvement des fluids," Mémoires de l'Académie des Sciences de L'Institut de France, vol. 6, pp. 389-440, 1823.

[10] I. J. Rao and K. R. Rajagopal, "The effect of the slip boundary condition on the flow of fluids in a channel," Acta Mechanica, vol. 135, no. 3-4, pp. 113-126, 1999.

[11] M. Mooney, "Explicit formulas for slip and fluidity," Journal of Rheology, vol. 2, no. 2, pp. 210-222, 1931.

[12] K. B. Migler, H. Hervet, and L. Leger, "Slip transition of a polymer melt under shear stress," Physical Review Letters, vol. 70, no. 3, pp. 287-290, 1993.

[13] S. Das, S. K. Guchhait, and R. N. Jana, "Hall effects on unsteady hydromagnetic flow past an accelerated porous plate in a rotating system," Journal of Applied Fluid Mechanics, vol. 8, no. 3, pp. 409-417, 2015.

[14] A. S. Chethan, G. N. Sekhar, and P. G. Siddheshwar, "Flow and heat transfer of an exponential stretching sheet in a viscoelastic liquid with navier slip boundary condition," Journal of Applied Fluid Mechanics, vol. 8, no. 2, pp. 223-229, 2015.

[15] M. Khan, R. Malik, and A. Anjum, "Exact solutions of MHD second Stokes flow of generalized Burgers fluid," Applied Mathematics and Mechanics, vol. 36, no. 2, pp. 211-224, 2015.

[16] I. Khan, F. Ali, and S. Shafie, "Stokes' second problem for magnetohydrodynamics flow in a Burgers' fluid: the cases $\gamma=\lambda^{2} / 4$ and $\gamma>\lambda^{2} / 4$," PLoS ONE, vol. 8, no. 5, Article ID e61531, 2013.

[17] A. M. Siddiqui, T. Haroon, M. Zahid, and A. Shahzad, "Effect of slip condition on unsteady flows of an oldroyd-B fluid between parallel plates," World Applied Sciences Journal, vol. 13, no. 11, pp. 2282-2287, 2011.

[18] D. Vieru and A. A. Zafar, "Some Couette flows of a Maxwell fluid with wall slip condition," Applied Mathematics \& Information Sciences, vol. 7, no. 1, pp. 209-219, 2013.
[19] D. Vieru and A. Rauf, "Stokes flows of a Maxwell fluid with wall slip condition," Canadian Journal of Physics, vol. 89, no. 10, pp. 1061-1071, 2011.

[20] C. Fetecau, N. Nigar, D. Vieru, and C. Fetecau, "First general solution for unidirectional motions of rate type fluids over an infinite plate," Communications in Numerical Analysis, vol. 2, pp. 125-138, 2015.

[21] C. Fetecau, D. Vieru, C. Fetecau, and I. Pop, "Slip effects on the unsteady radiative MHD free convection flow over a moving plate with mass diffusion and heat source," The European Physical Journal Plus, vol. 130, article 6, 2015.

[22] A.-R. A. Khaled and K. Vafai, "The effect of the slip condition on Stokes and Couette flows due to an oscillating wall: exact solutions," International Journal of Non-Linear Mechanics, vol. 39, no. 5, pp. 795-809, 2004.

[23] T. Hayat, M. F. Afzaal, C. Fetecau, and A. A. Hendi, "Slip effects on the oscillatory flow in a porous medium," Journal of Porous Media, vol. 14, no. 6, pp. 481-493, 2011.

[24] C. Fetecau, D. Vieru, C. Fetecau, and S. Akhter, "General solutions for magnetohydrodynamic natural convection flow with radiative heat transfer and slip condition over a moving plate," Zeitschrift für Naturforschung A, vol. 68, pp. 659-667, 2013. 


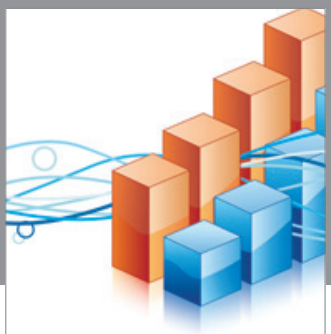

Advances in

Operations Research

vatem alat4



\section{The Scientific} World Journal
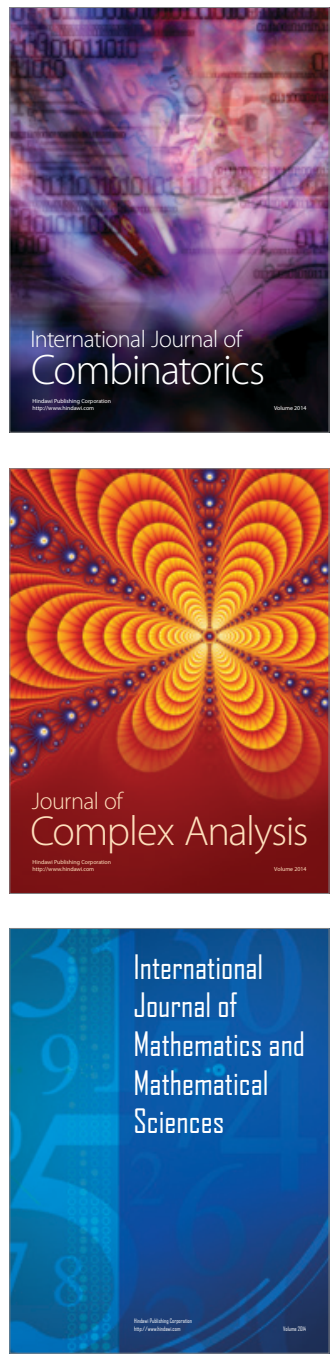
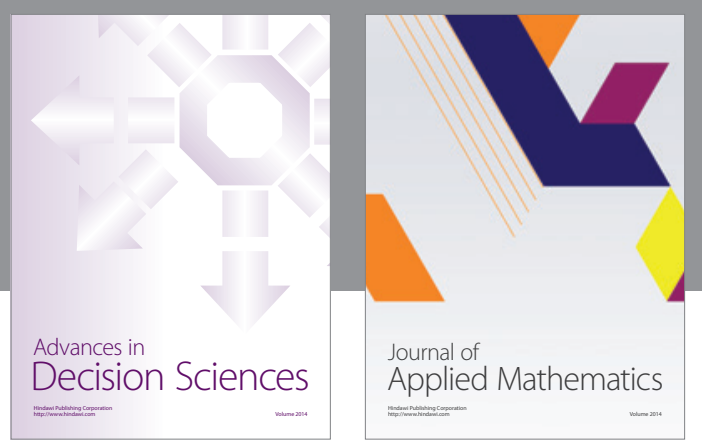

Algebra

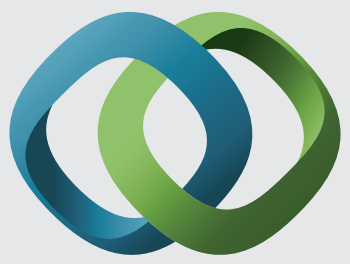

\section{Hindawi}

Submit your manuscripts at

http://www.hindawi.com
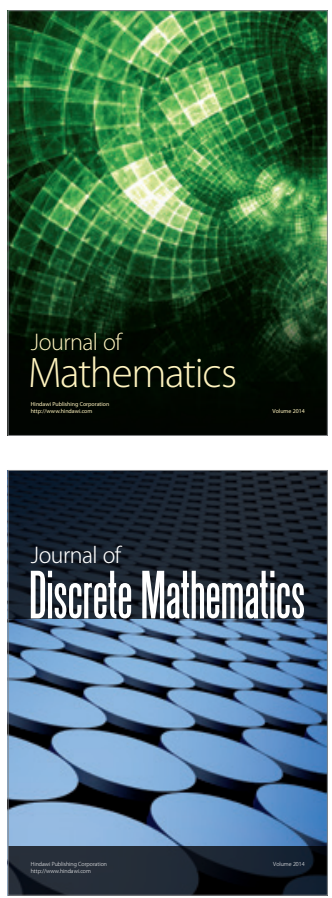

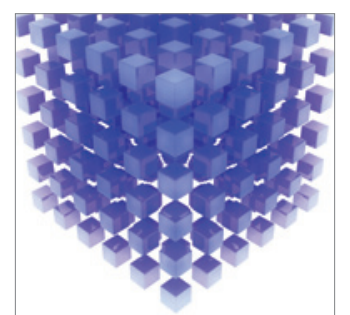

Mathematical Problems in Engineering


Journal of

Function Spaces

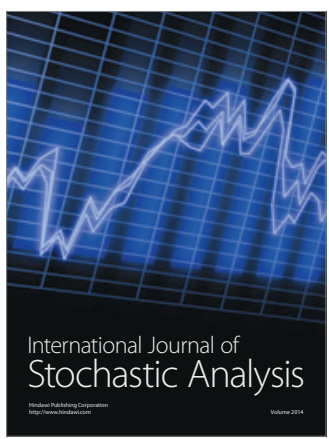

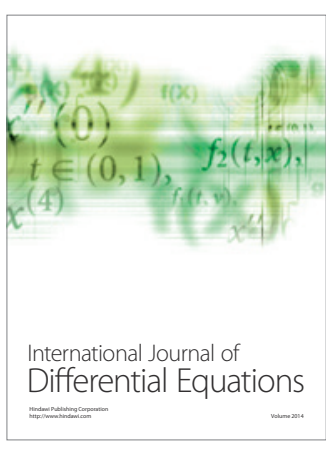
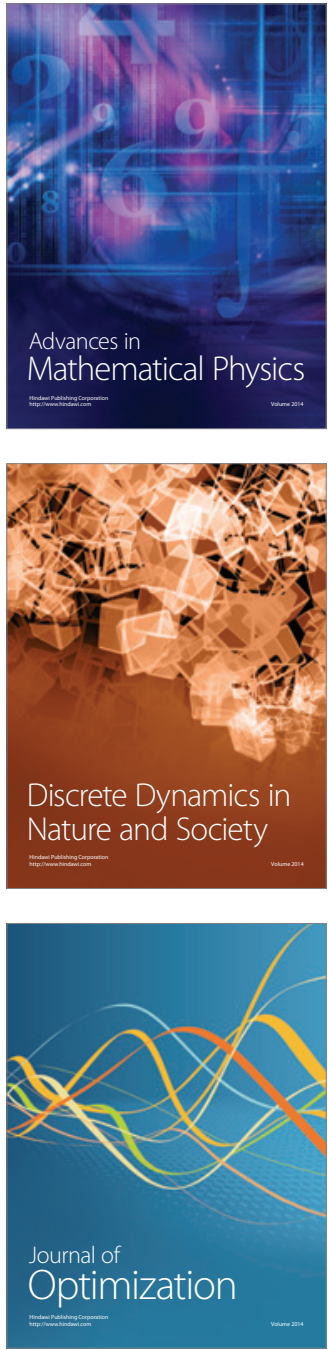